\title{
Accuracy of 2D Pulsatile Flow in the Lattice Boltzmann BGK Method
}

\author{
A. M. Artoli, A. G. Hoekstra, and P. M. A. Sloot \\ Section Computational Science, Informatics Institute, Faculty of Science \\ University of Amsterdam, Kruislaan 403, 1098 SJ Amsterdam, The Netherlands \\ \{artoli, alfons, sloot\}@science.uva.nl \\ http://www.science.uva.nl/research/scs/
}

\begin{abstract}
We present detailed analysis of the accuracy of the lattice Boltzmann BGK (LBGK) method in simulating oscillatory flow in a two dimensional channel. Flow parameters such as velocity and shear stress have been compared to the analytic solutions. Effects of different boundary conditions on the accuracy have been tested. When the flow is driven by a body force, we have observed a shift in time between the theory and the simulation, being about +0.5 , which when taken into account enhances the accuracy at least one order of magnitude.
\end{abstract}

\section{Introduction}

Recently, it has been demonstrated that the lattice-Boltzmann method (LBM) is useful in simulation of time-dependent fluid flow problems [1]. The method is attracting more attention for its inherent parallelism and straightforward implementation [2]. It is well known that, for steady flow, LBM is second order accurate in the velocity fields and the stress tensor when second order boundary conditions are applied [3],[4], [5]. Although the accuracy has been studied extensively for steady flow, studies on the accuracy of the LBM method for time dependent flows are quite rare [6].

Pulsatile flow characteristics are quite important in haemodynamics: Cardiovascular diseases are considered as the main causes of death in the world [7]. It is reasonably believed that the shear stress plays a dominant role in locality, diagnosis and treatment of such diseases [8]. With LBM, it is possible to compute the local components of the stress tensor without a need to estimate the velocity gradients from the velocity profiles [9], [5]. In addition, the equation of state defines the pressure as a linear function of the density which makes it quite simple to obtain the pressure from the density gradient. All these advantages makes the LBM suitable for simulating time-dependent flows.

In this article, we present the capability and investigate the accuracy of the lattice Boltzmann BGK (LBGK) model in recovering analytic solutions for oscillatory two dimensional (2D) channel flow in a range of Womersley and Reynolds numbers. We will first shortly review the model, then present the simulation results and conclude with a discussion. 


\section{The Lattice Boltzmann BGK Method}

Although the LBGK model historically evolved from the lattice gas automata, it can be derived directly from the simplified Boltzmann BGK equation [10] which mainly describes rarefied gases in the Boltzmann gas limit. This approximation works well for incompressible and isothermal flows. The method ensures isotropy and Galilean invariance. It is called LBGK since it simplifies the collision operator of the Boltzmann equation via the single particle relaxation time approximation proposed by Bhatnagar, Gross and Krook in 1954 [11]. The evolution equation of the distribution functions is [10]

$$
\frac{\partial f}{\partial t}+\boldsymbol{\xi} \cdot \nabla f=-\frac{1}{\lambda}(f-g)
$$

where $f \equiv f(x, \boldsymbol{\xi}, t)$ is the single particle distribution function, $\boldsymbol{\xi}$ is the microscopic velocity, $\lambda$ is the collision relaxation time and $g$ is the Maxwell-Boltzmann distribution function. The numeric solution for $f$ is obtained by discretizing Eq.(1) in the velocity space $\boldsymbol{\xi}$ using a finite set of velocities $\mathbf{e}_{i}$ without violating the conservation laws of the hydrodynamic moments. This gives [10]

$$
\frac{\partial f_{i}}{\partial t}+\mathbf{e}_{i} \cdot \nabla f_{i}=-\frac{1}{\lambda}\left(f_{i}-f_{i}^{(e q)}\right)
$$

where $f_{i}(\mathbf{x}, t) \equiv f\left(\mathbf{x}, \mathbf{e}_{i}, t\right)$ and $f_{i}^{(e q)}(\mathbf{x}, t)=f^{(0)}\left(\mathbf{x}, \mathbf{e}_{i}, t\right)$ are the distribution function and the equilibrium distribution function of $\mathbf{e}_{i}$, respectively. The equilibrium distribution function, which is the Taylor expansion of the Maxwellian distribution, takes the following form [12] in the limit of low Mach number:

$$
f_{i}^{(e q)}=w_{i}\left(\rho+\frac{3}{v^{2}} \mathbf{e}_{i} \cdot \mathbf{u}+\frac{9}{2 v^{4}}\left(\mathbf{e}_{i} \cdot \mathbf{u}\right)^{2}+\frac{3}{2 v^{2}} \mathbf{u} \cdot \mathbf{u}\right),
$$

where $w_{i}$ is a weighting factor, $v=\delta_{x} / \delta_{t}$ is the lattice speed, and $\delta_{x}$ and $\delta_{t}$ are the lattice spacing and the time step, respectively. The values of the weighting factor and the discrete velocities depend on the used LBM model and can be found in literature (see e.g. ref.[12]). In this study, we have used the improved incompressible D2Q9i model [12], which has three types of particles on each node; a rest particle, four particles moving along $x$ and $y$ principal directions with speeds $\left|\mathbf{e}_{i}\right|= \pm 1$, and four particles moving along diagonal directions with speeds $\left|\mathbf{e}_{i}\right|=\sqrt{2}$. The hydrodynamic density, $\rho$, and the macroscopic velocity, $\mathbf{u}$, are determined in terms of the particle distribution functions from

$$
\rho=\sum_{i} f_{i}=\sum_{i} f_{i}^{(e q)}
$$

and

$$
\rho \mathbf{u}=\sum_{i} \mathbf{e}_{i} f_{i}=\sum_{i} \mathbf{e}_{i} f_{i}^{(e q)}
$$


Equation (2) is then discretized in space and time into the well-known lattice BGK equation [6]

$$
f_{i}\left(\mathbf{x}+\mathbf{e}_{i} \delta_{t}, \mathbf{e}_{i}, t+\delta_{t}\right)-f_{i}\left(\mathbf{x}, \mathbf{e}_{i}, t\right)=-\frac{1}{\tau}\left[f_{i}\left(\mathbf{x}, \mathbf{e}_{i}, t\right)-f_{i}^{(0)}\left(\mathbf{x}, \mathbf{e}_{i}, t\right)\right]
$$

where $\tau=\frac{\lambda}{\delta_{t}}$ is the dimensionless relaxation time. Taylor expansion of Eq. (6) up to $O\left(\delta_{t}^{2}\right)$ and application of the multi-scale Chapman-Enskog technique [13] by expanding $f_{i}$ about $f_{i}^{(0)}$, we can derive the evolution equations and the momentum flux tensor up-to second order in the Knudsen number. The NavierStokes equation can be derived from this equation to yield

$$
\partial_{t} \mathbf{u}+(\mathbf{u} \cdot \nabla) \mathbf{u}=-\frac{1}{\rho} \nabla p+\nu \nabla^{2} \mathbf{u}
$$

where $p=\rho c_{s}^{2}$ is the scalar pressure and $\nu$ is the kinematic viscosity of the lattice Boltzmann model, given by

$$
\nu=c_{s}^{2} \delta_{t}\left(\tau-\frac{1}{2}\right)
$$

where $c_{s}$ is the speed of sound. Also, the stress tensor is

$$
\sigma_{\alpha \beta}=-\rho c_{s}^{2} \delta_{\alpha \beta}-\left(1-\frac{1}{2 \tau}\right) \sum_{i=0} f_{i}^{(1)} e_{i \alpha} e_{i \beta} .
$$

which can be computed directly during the collision process [5], without a need to compute the derivatives of the velocity fields. This extensively enhances the lattice Boltzmann BGK method, as other CFD methods are more elaborate and estimate the stress tensor components from the simulated velocity field. With this LBM model, the flow fields and the stress tensor components are accurate up to the second order in the Knudsen number. We emphasize that, with compressible LBM models, when the forcing term is time-dependent, compressibility effects may arise and using an incompressible model is a must [6]. However, since the D2Q9i model has already been tested for steady flows, for which it was proposed, we test it here for unsteady flows.

\section{Simulations}

We have conducted a number of $2 \mathrm{D}$ simulations for time dependent flow in a channel. Various boundary conditions have been tested: the Bounce-back on the nodes, the body force, periodic boundaries, non slip, and inlet and outlet boundary conditions. For all simulations described below, unless otherwise specified, the flow is assumed to be laminar, the Mach number is low, the driving force is the body force, the Reynolds number is defined as $R e=\frac{L^{3} G}{8 \nu^{2}}$ [3], the Womersley number is defined as $\alpha=\frac{L}{2} \sqrt{\frac{2 \pi}{\nu T}}$ [14] and the Strouhal number is defined as 
$S t=\frac{\alpha^{2}}{\pi R e}[7]$, where $L$ is the channel width, $\nu=\frac{2 \tau-1}{6}$ is the kinematic viscosity, $G$ is the body force, $T$ is the sampling period and $\omega=\frac{2 \pi}{T}$ is the angular frequency. The density is $\rho$ and the pressure gradient is sinusoidal with amplitude $A$ which is normalized to 1 by dividing by the magnitude of the body force when comparing simulation results to analytic solutions.

\subsection{Oscillatory channel flow}

We have studied the flow in an infinite 2D channel due to an oscillatory pressure gradient $\frac{\partial P}{\partial x}=A \sin (\omega t)$, where $A$ is a constant. The pressure gradient is implemented by applying an equivalent body force $G$. The analytic solution for the velocity in this case is given by[14]

$$
u(y, t)=R e\left[-\frac{A}{\rho \omega} e^{-i \omega t}\left(1-\frac{\cosh [\sqrt{b}(y-L / 2)]}{\cosh [\sqrt{b} L / 2]}\right)\right]
$$

where $\rho$ is the fluid density, $L$ is the width of the channel, and $b=-i \omega / \nu$. To check the accuracy of the LBGK model, we have performed a number of simulations. At first, we have used the bounce-back on the nodes which is quite simple and is known to be of second order accuracy[3]. The driving force for the flow is the body force $G$. Periodic boundary conditions are used for the inlet and the outlet boundaries. Both the Reynolds and the Womersley numbers were kept fixed by fixing the distance $L$ between the two plates and varying the relaxation parameter $\tau$, the period $T$ and the body force $G$. The error in velocity at each time step is defined by

$$
E v=\frac{\sum_{i=1}^{n}\left|u_{t h}-u_{l b}\right|}{\sum_{i=1}^{n} u_{t h}}
$$

where $u_{t h}$ is the analytic solution for the horizontal velocity, $u_{l b}$ is the velocity obtained from the LBGK simulations, $T$ is the period and $n$ is the number of lattice nodes representing the width of the channel. The overall error, Eav, is averaged over the period T. The relaxation time ranges from $\tau=0.6$ to $\tau=3$, the body force ranges from $G=25 \times 10^{-5}$ to $G=0.04$ and the sampling period lies in the range $500-20$, giving corresponding values of $0.2-5$ for $\delta t$, with $\delta_{t}=1$ corresponding to the case where $\tau=1$. The system was initialized by setting the velocity to zero everywhere in the system. The convergence criterion is attained by comparing simulation results from two successive periods and the stop criterion is when this difference is less than $10^{-7}$.

The agreement between the simulation and analytic solutions is quite good, as it is shown by the dashed lines in Fig. 1 which shows the velocity profiles for $\alpha=4.34$ for $t=0.75 T$. However, there seems to be a small shift in time between the simulation and the theory. This shift is a function of time and $\tau$. We have found that if we assume that the theory lags the simulation with a half time step, i.e $t_{l b}=t_{t h}+0.5$, the error reduces at least one order of 
magnitude for all $\tau$ values. Figure 1 shows a typical simulation result compared to the analytic solution with and without shifting the time coordinate. We have used this observation to compare the simulation results with the shifted analytic solution which leads to excellent agreement for all values of time, as shown in Fig. 2. The error behavior is shown in Fig. 3, from which it can be seen that the error is minimum at $\tau=1$, as expected, since there is no slip velocity at this specific case. An error of the order of the round-off error could be reached for the special case when $\tau=1$, when the bounce-back on the nodes is used [3], and assuming the 0.5 time shift.

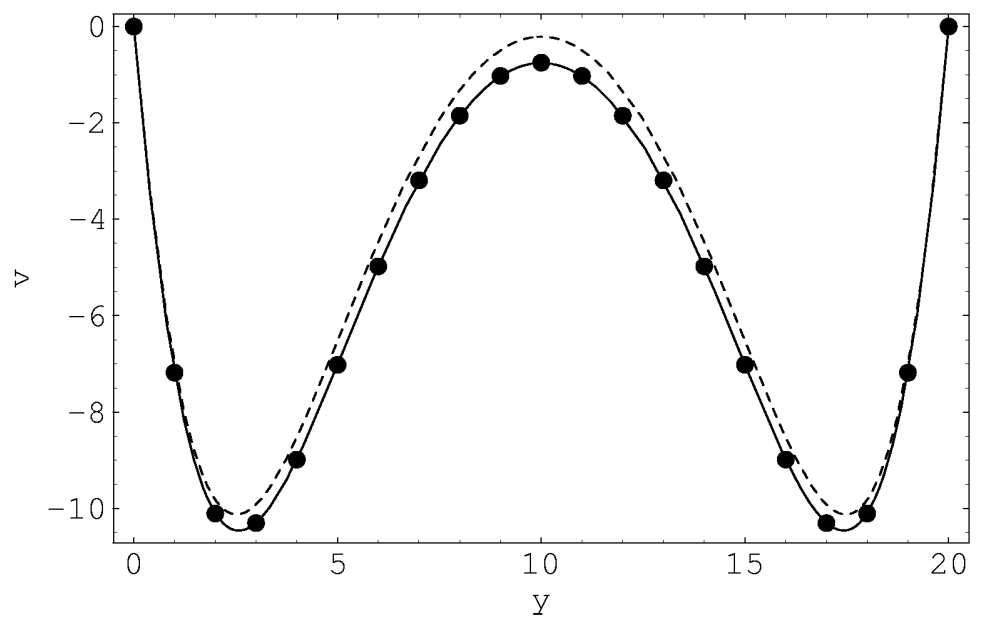

Fig. 1. Velocity profiles at $t=0.75 T$ with $\tau=1$ and $\alpha=4.34$ in a $2 \mathrm{D}$ oscillatory channel flow. The dots are the LBGK results. The dashed line is the analytic solution and the solid line is the analytic solution with a shift of 0.5 in time.

This shift in time has been observed before by Matthaeus [15]. It may be attributed to the way the driving force is imposed in the simulation and the used LBM model. We also believe that the way in which time coordinates are discretized may have some effects on this shift. For the other cases, when $\tau \neq 1$, the effect of the slip velocity dominates. Up to now, the slip velocity has analytic formulae for steady channel flow, but not yet for unsteady flow.

\subsection{Non slip boundaries}

In order to remove errors arising from the slip velocity, we have conducted similar simulations with a no-slip boundary condition [16] at the walls and periodic boundary conditions at the inflow and the outflow boundaries. The body force that corresponds to a desired Reynolds number drives the flow. The Strouhal 


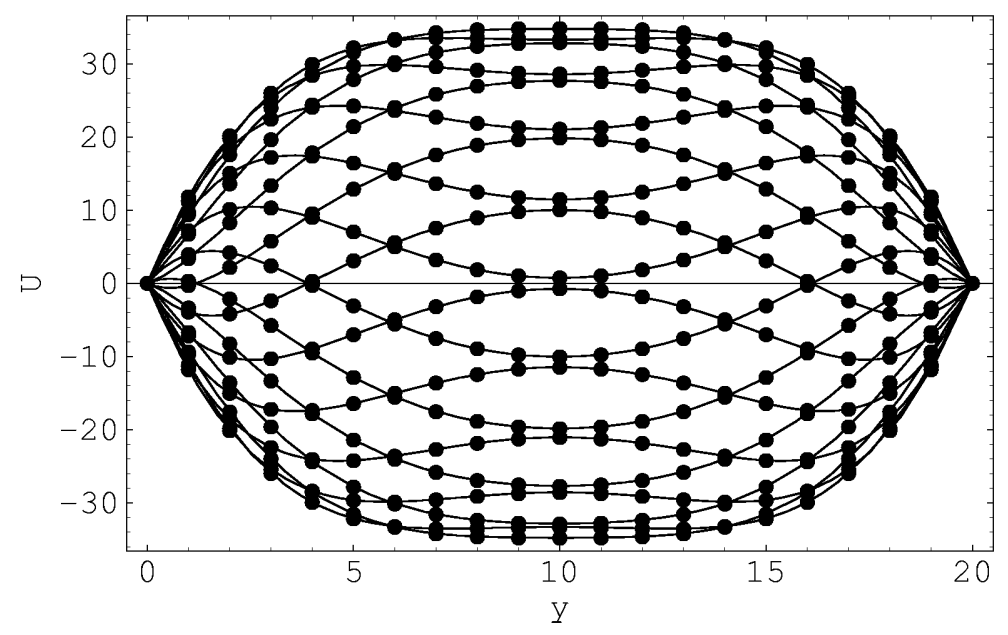

Fig. 2. Results from LBGK (dots) compared to the shifted theory (lines) for a complete period for $\alpha=4.34$ with $\tau=1$. The measurements are taken at the middle of the channel, at each $t=0.05 n T$ where $n=0,1, \ldots, 20$.

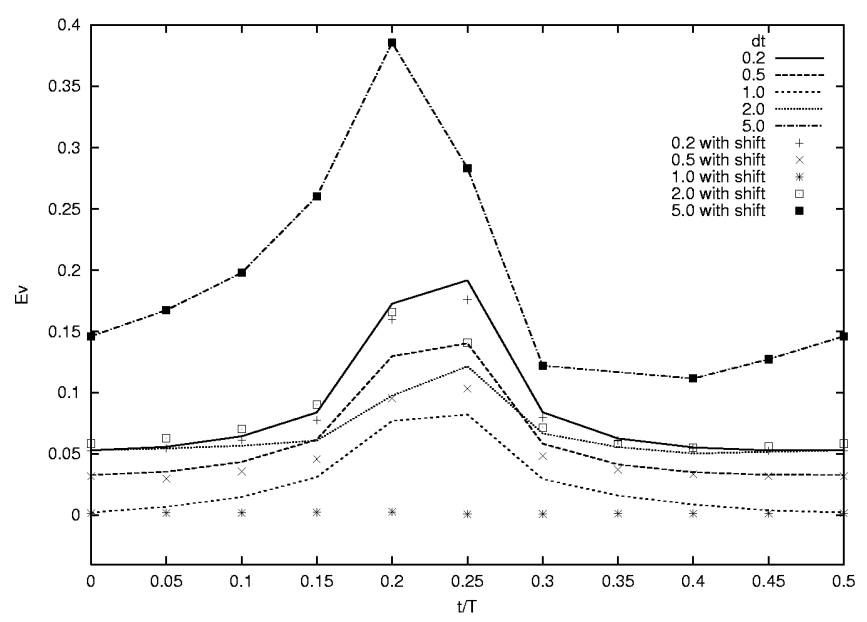

Fig. 3. Error behavior as a function of $\delta_{t}$ without (lines) and with (points) time-shift correction $\alpha=4.34$ in a $2 \mathrm{D}$ oscillatory tube flow 
number is kept constant by keeping fixed both the Reynolds and the Womersley numbers and looking at the accuracy in time. This is done by fixing the width $L$ and assigning the corresponding values for the sampling period $T$, the body force $G$, and the relaxation parameter $\tau$. In this way, $\delta_{t}$ will change. The results are shown in Fig. 4 which shows the error behavior as a function of $\delta_{t}$. From this figure, we clearly see that the LBM is of first order in time (slope $=0.9$ ). The error is again decreased when the half-time step correction is used, specially at $\tau=1$, as shown in the same graph. From this experiment, we conclude that the shift in time doesn't depend on the used boundary condition.

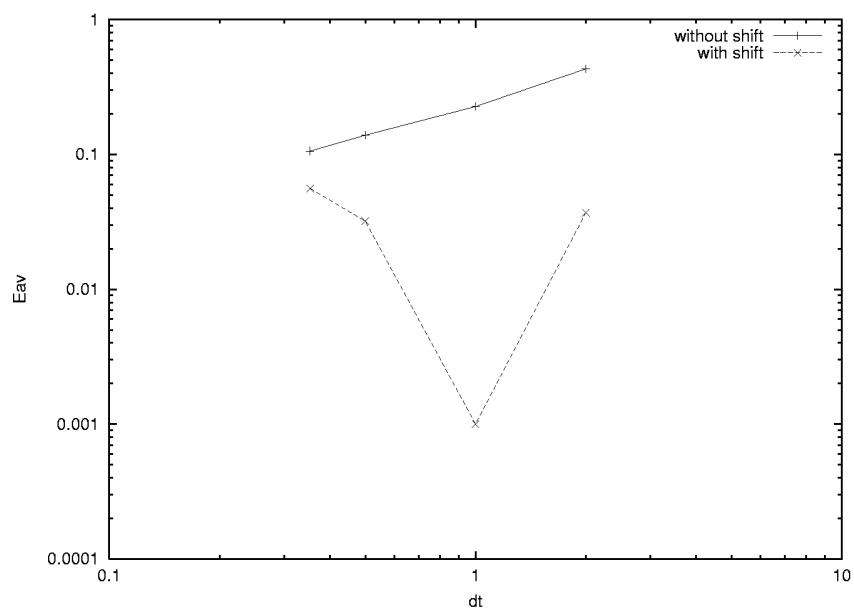

Fig. 4. Overall Relative Error of velocity vs $\delta_{t}$ for $\alpha=15.53$ and $R e=10$ in a $2 \mathrm{D}$ oscillatory channel flow. The slope of the straight line is 0.90 . The dashed line is the error with reference to the shifted in time analytic solution.

\subsection{Influence of the Reynolds number}

We have conducted another set of simulations to see the influence of the Reynolds number on the error in the flow fields. Here, the relaxation parameter is kept fixed at the value $\tau=1$, the width of the channel is varied to achieve higher Reynols numbers and the period is changed analogously to keep the Womersley parameter constant. The length of the channel is $5 L$. In summary, we change the Reynolds number $R e$, the body force $G$, the width $L$ and the period $T$. Simulations for Reynolds numbers in the range $1 \rightarrow 200$ at $\alpha=15.533$ were performed. Figure 5 shows comparisons of numerical and analytical solutions of the velocity profile for $R e=200$ at $t=0.2 T$. Similar agreements between theory and simulations has been observed for the whole period at different Reynolds numbers (data not shown). When compared to the analytic solutions, with and 
without shift in time, the error decreases from 0.0085 to 0.00024 for $R e=200$.

It can be seen that the difference between the two analytic solutions (the original and the shifted) becomes less as the Reynolds number increases. This suggests that the shift is inversely proportional to the applied body force which may have direct influence on it. It is therefore necessary to conduct another set of simulations in which the body force has no influence.

\subsection{Inlet and Outlet Pressure Boundary Conditions}

In order to remove the influence of the body force, we have conducted another set of simulations in which the flow in a $2 \mathrm{D}$ channel is driven with a sinusoidal pressure gradient of magnitude $A=0.001 / L_{x}$ where $L_{x}$ is the length of the channel. The length of the channel is 10 times the width and the period of the driving pressure is $T=1000$. The density at the inlet is $1+A \sin (\omega t)$ and is set to be 1.0 at the outlet. The convergence criterion is attained by comparing simulation results from two successive periods and the stop criterion is when this difference is less than $10^{-7}$. All the flow fields were initialized from zero. We have again observed excellent agreement with the theory, as it is shown in Fig. 6 . The shift in time has diminished in magnitude, but it is still there. From this experiment, we conclude that the main reason for this shift in time is due to an artifact in LBGK and is mainly attributed to incorrect implementation of the forcing term.

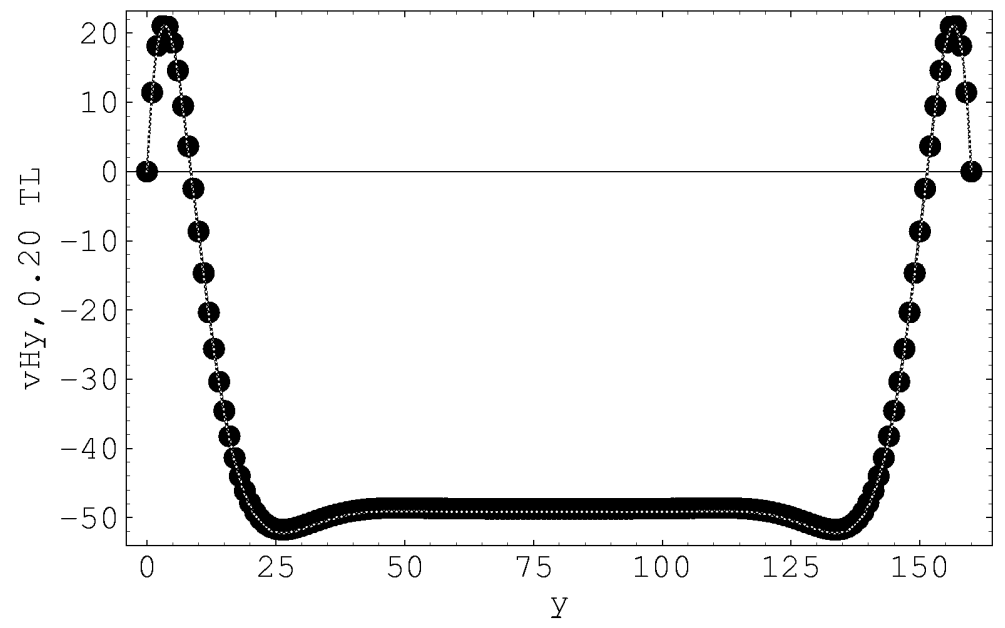

Fig. 5. Velocity profiles obtained from LBGK simulations (dots) for $\alpha=15.53$ and $R e=200$ in a $2 \mathrm{D}$ oscillatory channel flow, showing excellent agreement with the analytic solutions (lines). The effect of time shift is not observable. 


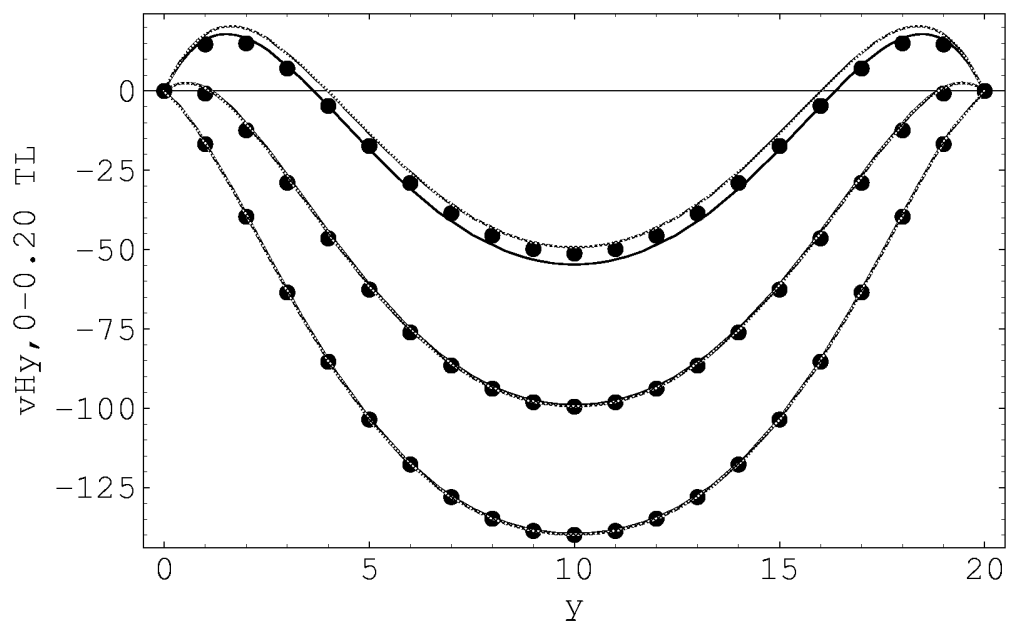

Fig. 6. Velocity profiles obtained from LBGK simulations (dots) for $\alpha=4.00017$ in a $2 \mathrm{D}$ oscillatory channel flow when inflow and outflow boundary conditions are used. Selected simulation times are shown. The shift in time (lines) has little effect but still there.

\subsection{Discussion and Conclusion}

We have shown that the lattice Boltzmann LBGK model can be used to simulate time dependent flows in 2D within acceptable accuracy if suitable simulation parameters and accurate boundary conditions are used. We have conducted a number of $2 \mathrm{D}$ simulations for time-dependent flow in a channel with different boundary conditions. We have observed a shift in time and have shown that the lattice Boltzmann BGK model is more accurate when a half time step correction is added to the time coordinates. We have investigated the time shift association with the used boundary conditions, and have found that it is always present for the cases we have studied. The effects of the Womersley, the Reynolds and the Strouhal numbers have also been studied. We also have looked at the accuracy in time for time-dependent flows and have shown that it is of first order, in agreement with the theory[10].

We have also measured the shear stress from the non-equilibrium parts of the distribution functions and have found good agreement with the analyticly derived stresses. However, it has been reported that LBGK is thermodynamically inconsistent and the forcing term leads to incorrect energy balance equation when the acceleration is not constant in space [17]. Therefore, it is argued that, it is better to use a general LBM model rather then the LBGK to overcome problems arising from artifacts in the LBGK model, since it is not suitable for dense gases. The modified Lattice Boltzmann method that is derived from the Enskog equation in which the $H$ theorems can be proved and the forcing term recovers correct energy balance equations [17]. However, on the other hand, the LBGK is simple and could work properly if being used cautiously. Currently, we have performed 
a number of three dimensional simulations using a quasi-incompressible model. The error is more than $12 \%$ with the simple bounce-back rule due mainly to the slip velocity and the artifacts in the LBKG model. Currently, we are working in enhancing the model and using more accurate boundary conditions. All this will be applied to simulations of a human pressure systolic cycle in order to be suitable for blood flow simulations of large arteries.

\section{References}

1. M. Krafczyk, M. Cerrolaza, M. Schulz, E. Rank, Analysis of 3D transient blood flow passing through an artificial aortic valve by Lattice-Boltzmann methods, $J$. Biomechanics 31, 453(1998).

2. D. Kandhai, A. Koponen, A. G. Hoekstra, M. Kataja, J. Timonen and P.M.Sloot, Lattice-Boltzmann hydrodynamics on parallel systems, Comp. Phys. Com. 111, 14 (1998).

3. X. He, Q. Zou, L. S. Luo, and M. Dembo. Analytical solutions of simple flow and analysis of non-slip boundary conditions for the lattice Boltzmann BGK model, $J$. of Stat. Phys., 87, 115 (1996).

4. S. Hou, Q. Zou, S. Chen, G. Doolen and A. C. Cogley. Simulation of cavity flow by the lattice-Boltzmann method, J.Comp. Phys. 118, 118 (1995).

5. A. M. Artoli, D. Kandhai, H. C. J. Hoefsloot, A. G. Hoekstra, and P. M. A. Sloot, Accuracy of the stress tensor in Lattice Boltzmann BGK Simulations, submitted to J. Comp. Phys.

6. Xiaoyi He and Li-Shi Luo, Lattice Boltzmann Model for the Incompressible NavierStokes Equation, J. Stat. Phys. 88927 (1997).

7. Y. C. Fung, Biomechanics:Circulation, 2nd edn., Springer, 192 (1997).

8. D. A. McDonald, Blood flow in Arteries, 2nd edn., The Camelot Press, (1974).

9. A. J. C. Ladd, Numerical simulations of particulate suspensions via a discretized Boltzmann equation. Part I. Theoretical foundation, J. Fluid Mech. 271285 (1994).

10. X. He and L. Luo, Theory of the lattice Boltzmann method: from the Boltzmann equation to the lattice Boltzmann equation, Phys. Rev. E, 56, 6811 (1997).

11. P. L. Bhatnagar, E. P. Gross, and M. Krook, A model for collision processes in gases. I. Small amplitude processes in charged and neutral one-component system, Phys. Rev. A, 94, 511 (1954).

12. Q. Zou, S. Hou, S.Chen and G.D. Doolen. An improved incompressible lattice Boltzmann model for time independent flows, J. Stat. Phys., October(1995).

13. B. Chopard and M. Droz, Cellular Automata modeling of Physical Systems, Cambridge University Press (1998).

14. C. Pozrikidis, Introduction to Theoretical and Computational Fluid Dynamics, OUP, (1997)

15. W. H. Mattheus, Bartol Research Institute, University of Delware, personal communication, International Conference on Discrete Simulation of Fluid dynamics, Cargese, July(2001).

16. Q. Zou and X. He, On pressure and velocity boundary conditions for the lattice Boltzmann BGK model, Phys. Fluids 9, 1591 (1997).

17. Li-Shi Luo, Theory of the lattice Boltzmann method: Lattice Boltzmann models for non-ideal gases, Phys.Rev. E 62, 4982(2000). 